Journal for ImmunoTherapy of Cancer

\title{
Toripalimab plus intensity-modulated radiotherapy for recurrent nasopharyngeal carcinoma: an open- label single-arm, phase II trial
}

\author{
Yijun Hua, ${ }^{1,2}$ Rui You (D) , ${ }^{1,2}$ Zhiqiang Wang, ${ }^{1,2,3}$ Peiyu Huang, ${ }^{1,2}$ Mei Lin (D) , ${ }^{1,2}$ \\ Yanfeng Ouyang, ${ }^{1,2}$ Yulong Xie, ${ }^{1,2}$ Xiong Zou, ${ }^{1,2}$ Youping Liu, ${ }^{1,2,4}$ Chongyang Duan, ${ }^{5}$ \\ Yonglong Liu, ${ }^{1,2}$ Chenmei Gu, ${ }^{1,2}$ Rongzeng Liu, ${ }^{1,2}$ Qi Yang, ${ }^{1,2}$ Rou Jiang, ${ }^{1,2}$ \\ Mengxia Zhang, ${ }^{1,2}$ Xi Ding, ${ }^{1,2}$ Siyuan Chen, ${ }^{1,2}$ Chao Lin, ${ }^{1,2}$ Rui Sun, ${ }^{1,2}$ \\ Mingyuan Chen ${ }^{1,2,4}$
}

To cite: Hua Y, You R, Wang Z, et al. Toripalimab plus intensitymodulated radiotherapy for recurrent nasopharyngeal carcinoma: an open-label single-arm, phase II trial. Journal for ImmunoTherapy of Cancer 2021;9:e003290. doi:10.1136/jitc-2021-003290

- Additional supplemental material is published online only. To view, please visit the journal online (http://dx.doi.org/10. 1136/jitc-2021-003290).

$\mathrm{YH}, \mathrm{RY}, \mathrm{ZW}, \mathrm{PH}, \mathrm{ML}$ and $\mathrm{YO}$ contributed equally.

Accepted 01 October 2021

Check for updates

(C) Author(s) (or their employer(s)) 2021. Re-use permitted under CC BY-NC. No commercial re-use. See rights and permissions. Published by BMJ.

For numbered affiliations see end of article.

Correspondence to

Mingyuan Chen;

chenmy@sysucc.org.cn

\section{ABSTRACT}

Background Toripalimab is a humanized immunoglobulin $\mathrm{G}_{4}$ monoclonal antibody against programmed death 1 . We aimed to investigate the efficacy and safety of toripalimab in combination with intensity-modulated radiotherapy (IMRT) for recurrent nasopharyngeal carcinoma (rNPC). Methods We conducted a single-arm, phase II trial with patients with rNPC who had biopsy-proven disease and were unsuitable for local surgery. Eligible patients received IMRT in combination with toripalimab administered via intravenous infusion of $240 \mathrm{mg}$ once every 3 weeks for a maximum of seven cycles. The primary endpoint was the objective response rate at 3 months post radiotherapy. The secondary endpoints included safety profiles, progressionfree survival (PFS).

Results Between May 2019 and January 2020, a total of 25 patients with rNPC were enrolled (18 men (72.0\%) and 7 women (28.0\%); median (IQR) age, 49.0 (43.5-52.5) years). With a median (IQR) follow-up duration of 14.6 months (13.1-16.2) months, 19 patients (79.2\%) achieved an overall response, and disease control was achieved in $23(95.8 \%)$ patients at 3 months post radiotherapy. The 12-month PFS was $91.8 \%$ (95\% Cl $91.7 \%$ to $91.9 \%$ ). The incidences of acute (grade $\geq 3$ ) blood triglyceride elevation, creatine kinase elevation, skin reaction, and mucositis were $1(4.0 \%), 1(4.0 \%), 2(8.0 \%)$, and $1(4.0 \%)$, respectively. The incidences of late severe (grade $\geq 3$ ) nasopharyngeal wall necrosis, nasal bleeding, and trismus were $28.0 \%, 12.0 \%$, and $4.0 \%$, respectively.

Conclusions Toripalimab combined with IMRT was tolerable and showed promising antitumor activity in patients with rNPC.

Trial registration number NCT03854838.

\section{INTRODUCTION}

Nasopharyngeal carcinoma (NPG) has the highest prevalence in Southeastern Asia, with age-standardized rates ranging from 22.2 to 27.2 per 100,000 among men. ${ }^{1}$ The incidences of local recurrence in endemic NPC range from $10 \%$ to $20 \%$ after previous radical radiotherapy. ${ }^{23}$ In this subgroup of patients with resectable diseases, surgery is the standard of care and first-line treatment. ${ }^{4}$ However, reirradiation is routinely performed in patients with unresectable lesions. Despite the utilization of intensity-modulated radiotherapy (IMRT) and combined chemotherapy, the response rate was reported to be approximately $50 \%-65.4 \%,{ }^{6}$ and the 3 -year overall survival (OS) rate was reported to be only $41.8 \%-68.7 \%$, with high rates of grade 5 treatment-related toxicities of approximately $33.0 \% .{ }^{78}$ Therefore, there is an urgent need to develop new therapies combined with IMRT in unresectable recurrent NPC (rNPC) that potentially reduce toxicity and improve survival benefit.

Immune checkpoint inhibitors against programmed death 1 (PD-1), exemplified by the humanized monoclonal antibodies pembrolizumab and nivolumab, have been approved for the treatment of patients with relapsed squamous cell carcinoma of the head and neck (HNSCC) after previous platinumbased therapy. Recently, the promising antitumor activity and good tolerance of PD-1 therapies including nivolumab, pembrolizumab, toripalimab and camrelizumab were reported in several phase $1 / 2$ trials for recurrent or metastatic NPC; however, the efficacy of PD-1 therapy alone was moderate, with a reported objective response rate of $20 \%-30 \% .{ }^{9-11}$ Theoretically, radiotherapy can improve the efficacy of tumor immunotherapy by modulating the peptide repertoire and enhancing major histocompatibility complex (MHC) I expression, ${ }^{12}{ }^{13}$ which reveals the rationale behind combining radiotherapy and PD-1 therapy. Recently, two 


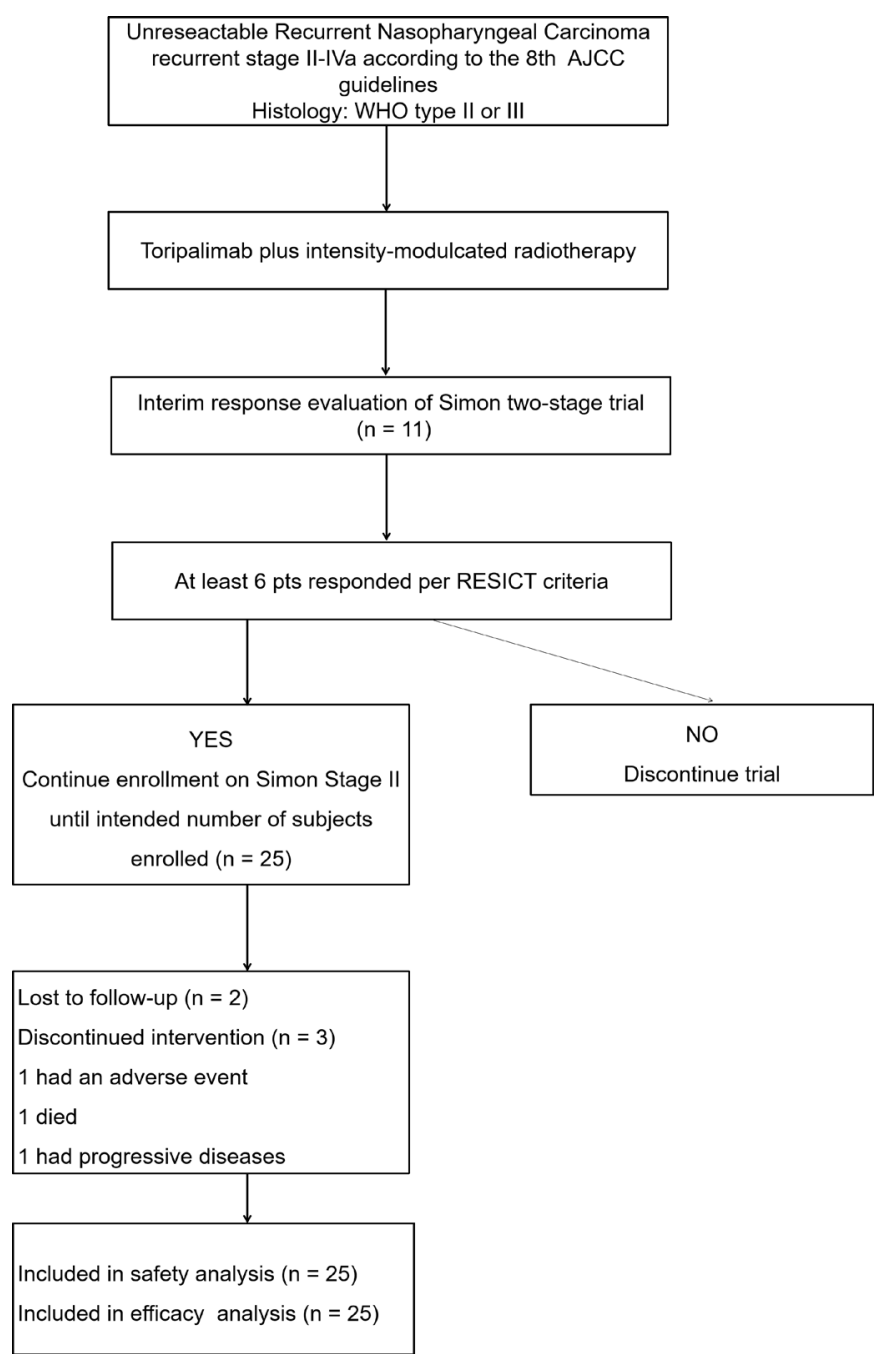

Figure 1 Trial profile. RECIST, Response Evaluation Criteria in Solid Tumors. AJCC, American Joint Committee on Cancer.

phase 1 studies demonstrated the safety and promising efficacy of pembrolizumab in combination with chemoradiotherapy in locally advanced HNSCC and non-small cell lung cancer. ${ }^{14}{ }^{15}$ However, there are still no reports of radiotherapy combined with PD-1 therapy in NPC.

We therefore conducted a phase 2, Simon two-stage clinical trial investigating the efficacy and safety of toripalimab, a humanized immunoglobulin $\mathrm{G}_{4}$ monoclonal antibody against PD-1, in combination with IMRT in rNPC.

\section{PATIENTS AND METHODS \\ Study design}

This report presents the analysis of a single-arm, phase 2 trial to assess the preliminary antitumor activity and safety of toripalimab combined with IMRT as the first-line therapy for patients with unresectable locally rNPC. The trial was performed in accordance with the Declaration of Helsinki and Good Clinical Practice guidelines. All participants provided written informed consent. The trial was registered at ClinicalTrials.gov.

\section{Patient selection}

The eligibility criteria for this trial included age 18-65 years; histologically confirmed NPC; patients with local recurrence (recurrent II-IVa) after curative radiotherapy who are unsuitable for local surgery; recurrence interval more than 12 months from the end of prior radiotherapy; no previous systemic chemotherapy for recurrent disease; an Eastern Cooperative Oncology Group performance status of 0 or 1; at least one measurable lesion assessed with Response Evaluation Criteria in Solid Tumors V.1.1 (RECIST V.1.1) by the investigators; and adequate organ function. Exclusion criteria included a history of active autoimmune disease; medical conditions requiring the use of immunosuppressive medications; active hepatitis $\mathrm{B}$ or $\mathrm{C}$ virus infection; uncontrolled hypertension and cardiac disease; previous treatment with anti-PD-1 or antiprogrammed death-ligand 1 (PD-L1) antibodies; and patients who were pregnant or breast feeding.

Pretreatment evaluation included a complete medical history and physical examination; hematologic and biochemical analyses; nasopharyngoscopy; and MRI or contrast-enhanced CT if patients had contraindications to MRI of the head and neck. ${ }^{18}$ F-fluorodeoxyglucose positron emission tomography was utilized to exclude distant metastasis.

\section{Procedures}

Toripalimab was administered via intravenous infusion of $240 \mathrm{mg}$ once every 3 weeks for a maximum of seven cycles or until disease progression, death, or doselimiting toxicities occurred or the patient requested to stop treatment. Off-protocol anticancer drugs were not allowed before the occurrence of protocol-defined disease progression. Dose modifications of toripalimab were not permitted. Details of the interruption and discontinuation of toripalimab are provided in the online supplemental file, Protocol.

Patients received IMRT after the first cycle of toripalimab. IMRT target volumes were delineated according to a previously described institutional treatment protocol. Briefly, the primary NPC tumor (gross tumor volume (GTV) nx) and gross cervical lymph nodes (GTVnd) were delineated. The clinical tumor volume (CTV) was then defined by GTVnx with a $0.5-1.0 \mathrm{~cm}$ margin. The prescribed doses were $60 \mathrm{~Gy}, 60 \mathrm{~Gy}$ and $54 \mathrm{~Gy}$ in 27 fractions for the planning target volumes derived from GTVnx, GTVnd, and CTV, respectively. Complete details on the radiotherapy planning are provided in the online supplemental appendix.

Tumor response after radiotherapy was based on the RECIST criteria V.1.1 and assessed by nasopharyngoscopy and MRI of the primary site. We defined a complete response (CR) as a complete lack of unequivocal soft tissue mass in the local region and cervical lymph nodes 
Table 1 Baseline demographics and disease characteristics

\begin{tabular}{ll}
\hline Characteristic & $\begin{array}{l}\text { Toripalimab plus } \\
\text { IMRT } \\
\mathbf{N}=\mathbf{2 5}\end{array}$ \\
\hline Sex & \\
Female & $7(28.0 \%)$ \\
Male & $18(72.0 \%)$
\end{tabular}

Age, years

Median 49.0

IQR

$43.5-52.5$

Karnofsky performance status score

90-100

$70-80$

$25(100.0 \%)$

$0(0.0 \%)$

Smoking status

$\begin{array}{ll}\text { Smokers } & 2(8.0 \%) \\ \text { Non-smokers } & 23(92.0 \%)\end{array}$

Histology

Non-keratinizing undifferentiated 23 (92.0\%)

(type III)

Non-keratinizing differentiated $\quad 2(8.0 \%)$

(type II)

EBV DNA copy number

$\begin{array}{ll}\text { Median } & 77.5 \\ \text { IQR } & 0-382.5\end{array}$

\section{Primary T classification}

\begin{tabular}{ll} 
T1 & $3(12.0 \%)$ \\
T2 & $6(24.0 \%)$ \\
T3 & $9(36.0 \%)$ \\
T4 & $7(28.0 \%)$ \\
\hline
\end{tabular}

Primary $\mathrm{N}$ classification

$\begin{array}{cl}\text { N0 } & 1(4.0 \%) \\ \text { N1 } & 7(28.0 \%) \\ \text { N2 } & 16(64.0 \%) \\ \text { N3 } & 1(4.0 \%) \\ \text { Primary M classification } & \\ \text { M0 } & 25(100.0 \%) \\ \text { M1 } & 0(0.0 \%)\end{array}$

Chemotherapy during primary

therapy

\begin{tabular}{cl} 
Yes & $24(96.0 \%)$ \\
No & $1(4.0 \%)$ \\
Recurrent T classification & \\
T2 & $2(8.0 \%)$ \\
T3 & $16(64.0 \%)$ \\
T4 & $7(28.0 \%)$ \\
Recurrent N classification & \\
N0 & $16(64.0 \%)$ \\
\hline
\end{tabular}

Continued
Table 1 Continued

\begin{tabular}{ll}
\hline Characteristic & $\begin{array}{l}\text { Toripalimab plus } \\
\text { IMRT } \\
\text { N=25 }\end{array}$ \\
\hline $\begin{array}{l}\text { N1 } \\
\text { Recurrent M classification }\end{array}$ & $9(36.0 \%)$ \\
M0 & $25(100.0 \%)$ \\
M1 & $0(0.0 \%)$ \\
Recurrent stage & $2(8.0 \%)$ \\
II & $16(64.0 \%)$ \\
III & $7(28.0 \%)$ \\
IVa & $17(68.0 \%)$ \\
PRANCIS prognostic index & $8(32.0 \%)$ \\
Low risk & $37.0(21.5-73.0)$ \\
High risk & \\
Disease-free interval, median \\
months (IQR) \\
Previous radiotherapy \\
$\begin{array}{l}\text { No } \\
\text { Yes }\end{array}$ \\
\hline
\end{tabular}

Data are presented as the median or $\mathrm{n}(\%)$ unless otherwise stated. EBV, Epstein-Barr virus; IMRT, intensity-modulated radiotherapy ; PRANCIS, Predicting Radioresistant Nasopharynx Carcinoma Survival.

that all had a short axis of less than $10 \mathrm{~mm}$ according to the RECIST guidelines. ${ }^{16}$

Patients were followed up after the completion of radiotherapy every 3 months until death to evaluate the efficacy and safety of the treatment. We recorded the survival status and subsequent lines of therapies. Adverse events (AEs) were scored according to the Common Terminology Criteria for Adverse Events V.5.0 and the acute and late radiation morbidity scoring criteria of the Radiation Therapy Oncology Group at each follow-up visit. Acute AEs were defined as those occurred during the radiotherapy or within 3 months post radiotherapy, while late AEs were defined as those occurred over 3 months after the end of radiotherapy.

\section{Study endpoints}

The primary endpoint of the study was the objective response rate (ORR) at 3 months after radiotherapy, which was defined as the proportion of patients who had a confirmed objective response (defined as complete or partial response according to RECIST V.1.1). The secondary endpoints included safety profiles, progression-free survival (PFS) (defined as the time from enrollment to locoregional or distant metastasis relapse or death from any cause, whichever occurred first), OS (defined as interval from enrollment to death due to any cause) and biomarker analysis. Imaging results to assess the ORR and PFS were centrally reviewed. 
Table 2 Response and survival data

Toripalimab plus IMRT

\begin{tabular}{|c|c|}
\hline $\begin{array}{l}\text { Overall response at } 3 \text { months post } \\
\text { radiotherapy* }\end{array}$ & $\mathrm{N}=24$ \\
\hline Overall response & $\begin{array}{l}19(79.2 \% ; 95 \% \mathrm{Cl} \\
59.5 \% \text { to } 90.8 \%)\end{array}$ \\
\hline Disease control & $23(95.8 \%)$ \\
\hline Complete response & $11(45.8 \%)$ \\
\hline Partial response & $8(33.3 \%)$ \\
\hline Stable disease & $4(16.7 \%)$ \\
\hline Progressive disease & $1(4.2 \%)$ \\
\hline $\begin{array}{l}\text { Best overall response after } \\
\text { radiotherapy }\end{array}$ & $\mathrm{N}=25$ \\
\hline Overall response & $\begin{array}{l}23(92.0 \% ; 95 \% \mathrm{Cl} \\
75.0 \% \text { to } 97.8 \%)\end{array}$ \\
\hline Disease control & $25(100.0 \%)$ \\
\hline Complete response & $18(72.0 \%)$ \\
\hline Partial response & $5(20.0 \%)$ \\
\hline Stable disease & $2(8.0 \%)$ \\
\hline Progressive disease & $0(0.0 \%)$ \\
\hline Time to response, months & $2.65(1.88-5.27)$ \\
\hline $\begin{array}{l}\text { Median duration of response, } \\
\text { months (IQR) }\end{array}$ & NR (NR-NR) \\
\hline \multicolumn{2}{|l|}{ Progression-free survival (PFS) } \\
\hline Failure & 5 \\
\hline Median PFS, months $(95 \% \mathrm{Cl})$ & 18.67 (12.04 to 25.29$)$ \\
\hline PFS rate at 12 months $(95 \% \mathrm{Cl})$ & $91.8 \%(91.7 \%$ to $91.9 \%)$ \\
\hline
\end{tabular}

*As there was one patient who did not undergo radiology examination for tumor response evaluation at 3 months post radiotherapy, a total of 24 patients were included in the analysis of overall response at 3 months post radiotherapy. IMRT, intensity-modulated radiotherapy .

\section{Exploratory studies}

Tumor biopsy samples were obtained from patients before treatment initiation. PD-L1 expression was detected by immunohistochemistry (IHC) using the antibody 22C3 pharmDx assay (Dako, Agilent Technologies, Santa Clara, California). PD-L1 expression was evaluated on tumor cells and on tumor-infiltrating immune cells by certified pathologists. PD-L1-positive status was defined as the presence of membrane staining of any intensity in $1 \%$ or more tumor cells or the presence of PD-L1 staining of any intensity in tumor-infiltrating immune cells covering $1 \%$ or more of the tumor area occupied by tumor cells, associated intratumoral cells, and contiguous peritumoral stroma. ${ }^{17}$

All tumor samples for sequencing were histologically confirmed as NPC and obtained at enrollment at Sun Yat-sen University Cancer Center (Guangzhou, China). Eighteen qualified primary nasopharyngeal lesions were obtained for subsequent whole-exome sequencing (WES), among which 7 primary lesions were formalin-fixed and parrffin-embedded (FFPE) samples and 11 were fresh frozen tissues. WES was performed using the Sure-Select Human All Exon V.6 Kit (Agilent) on tumor biopsies and matched peripheral blood mononuclear cell samples. Genomic alterations including single nucleotide variants, short and long insertions/deletions (indels), copy number variants, and gene fusions were assessed. The tumor mutation burden (TMB), frameshift indels, neoantigen burden, copy number burden-the weighted Genome Integrity Index (wGII) score and microsatellite instability score were determined.

\section{Statistical planning and analysis}

A Simon two-stage optimal design with a one-sided type I error rate of $5 \%$ and power of $80 \%$ was utilized. The null hypothesis was an ORR at 3 months post radiotherapy of $\leq 50 \%$, and the alternate hypothesis was an ORR $\geq 75 \%$. Consequently, 11 subjects were enrolled in the first stage. If six or fewer responded at the initial stage, the trial would be terminated, and the study would be concluded. If more than 6 patients achieved a partial or CR, then the treatment would be considered worthy of further investigation, and 14 more subjects would be enrolled in the second stage for a total sample size of 25 subjects. If there were more than 16 subjects with partial or $\mathrm{CR}$, then the treatment regimen was considered a success.

All enrolled patients were included in the efficacy and safety analyses. For all patients, the median follow-up time was calculated using the reverse KaplanMeier method. The ORR and 95\% CIs were calculated using the Clopper-Pearson method. The duration of response, PFS, and OS were analyzed using the KaplanMeier method. Summary statistics were provided for clinical and demographic characteristics and for AEs.

In post-hoc analyses, the associations between PD-L1 expression, genome/clinical characteristics and CR and PFS were assessed. For the comparison between patients with CR or not, $\chi^{2}$ test or Fisher's exact test and Wilcoxon rank-sum test were used. Univariate analysis of the effects of these parameters on PFS was conducted by the Cox proportional hazards model to calculate the HRs and 95\% CIs. We performed all statistical tests using Stata V.14.2 software (StataCorp). The authenticity of this article has been validated by uploading the key raw data onto the Research Data Deposit public platform (www.researchdata.org.cn), with the approval RDD number RDDA2021851016.

\section{RESULTS}

\section{Patients and treatment}

Between May 2019 and January 2020, a total of 25 patients with rNPC were enrolled in the study. All 25 patients' lesions were recurrent in the irradiation field. All 25 patients were included in the efficacy and safety analysis (figure 1). 
A

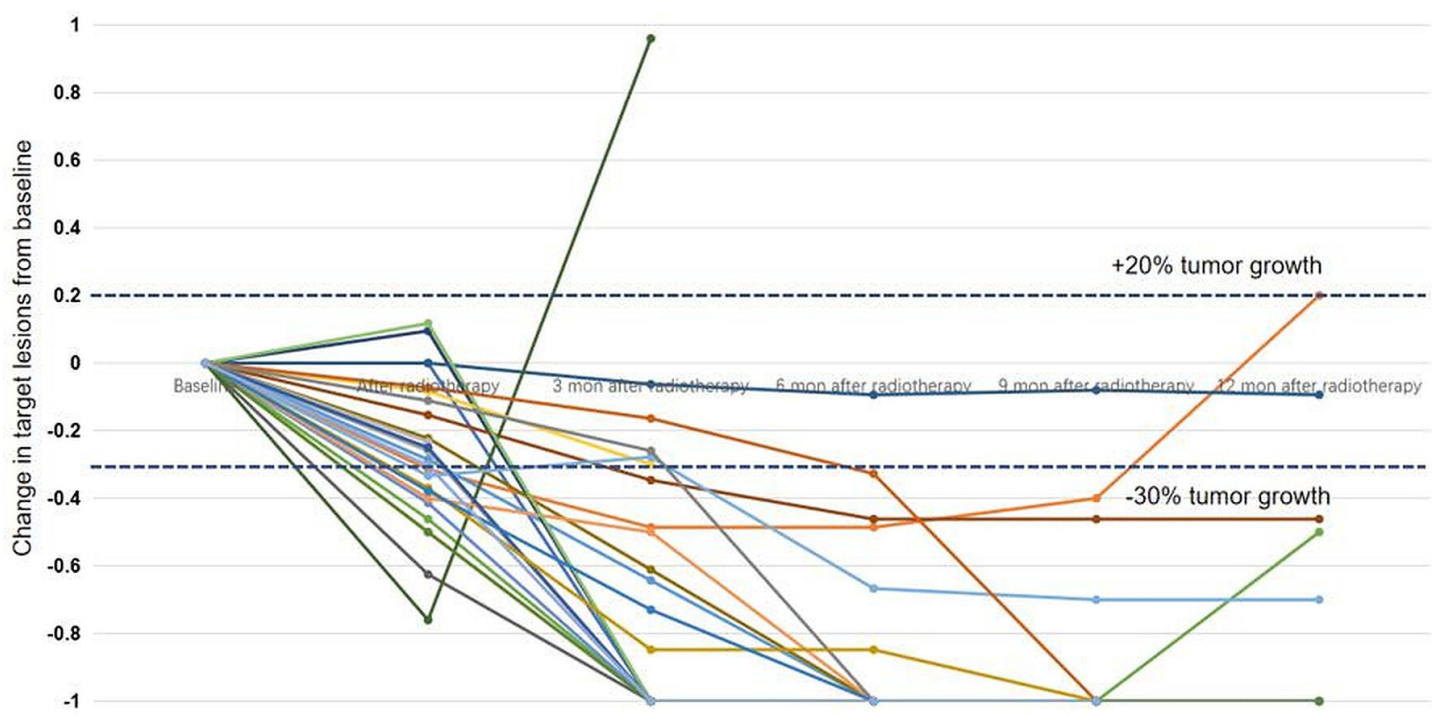

B

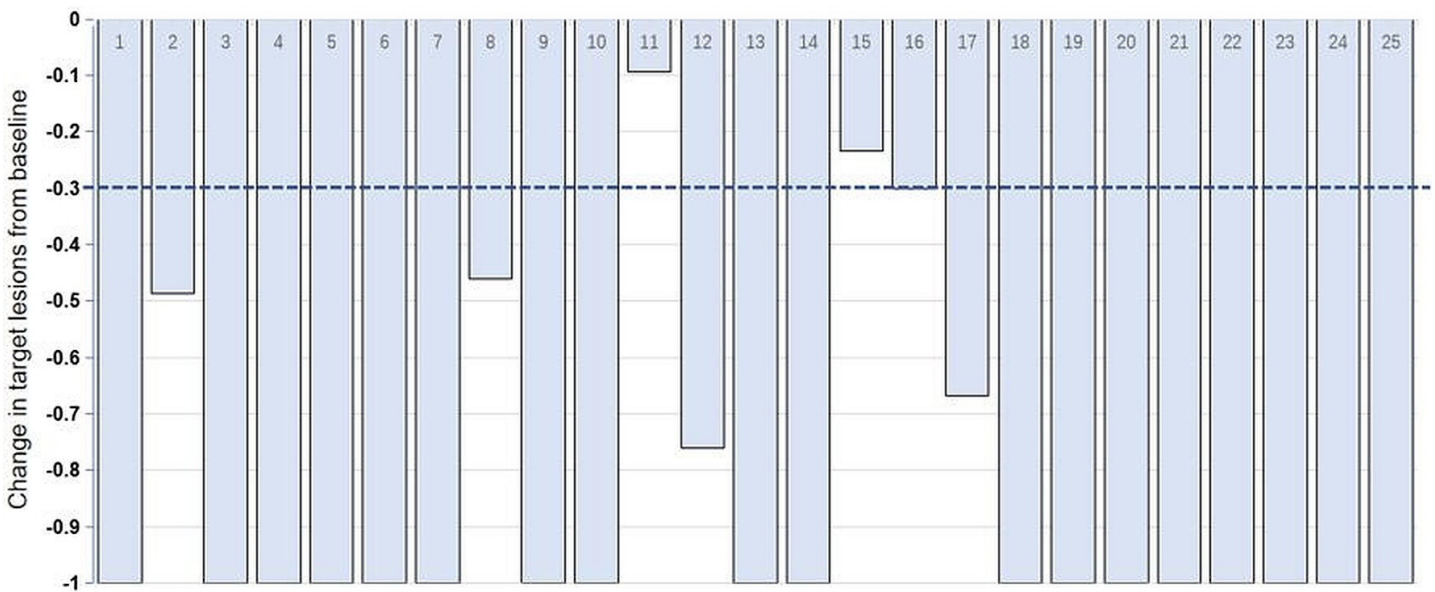

Figure 2 Antitumor activity. (A) Change in individual tumor burden during the first 12 months after the completion of radiotherapy from baseline assessed by Investigators per RECIST V.1.1 (N=25); (B) maximal change in tumor size from baseline assessed by investigators per RECIST V.1.1 ( $\mathrm{N}=25)$. The length of the bar represents the maximal decrease or minimal increase in target lesions. RECIST, Response Evaluation Criteria in Solid Tumors.

The baseline demographics and disease characteristics are listed in table 1. The median age was 49.0 (IQR 43.5$52.5)$ years. Twenty-three of $25(92.0 \%)$ patients were stage rT3-T4, and 25 of $25(100.0 \%)$ patients were stage rN0-N1. Seventeen of $25(68.0 \%)$ patients were defined as low risk, while 8 of $25(32.0 \%)$ patients were defined as high risk according to Predicting Radioresistant Nasopharynx Carcinoma Survival (PRANCIS) prognostic model. $^{2}$ Twenty-four of the 25 patients $(96.0 \%)$ were treated with platinum-based chemotherapy during their primary therapy. Twenty-two of the 25 patients $(88.0 \%)$ completed all seven cycles of toripalimab; one patient received only five cycles because of death from hemorrhage. Two patients received six cycles (one patient developed disease progression, and one patient discontinued treatment due to AEs). No modifications of the dose of toripalimab were observed. Toripalimab administration was delayed in one patient due to the COVID-19 pandemic. All 25 patients $(100.0 \%)$ completed the protocol-defined IMRT. The median dose of IMRT was 60.21 Gy (IQR, 60-60.21 Gy), and the median duration of IMRT was 36 days (IQR, 35-38 days) (online supplemental table 1).

\section{Efficacy}

In the first 11 patients enrolled, confirmed responses were noted in 10 patients. The ORR threshold for the first stage of Simon's two-stage was reached, and the trial continued to full accrual. The data cut-off date for the analysis was February 2021. Two patients were lost to follow-up, 


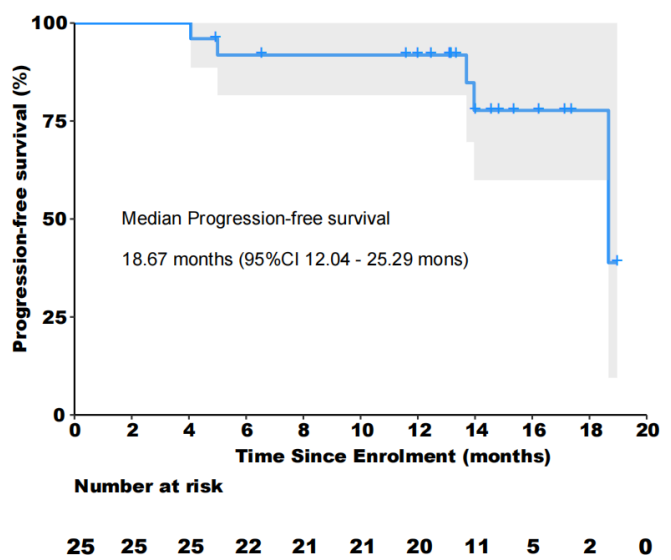

Figure 3 Kaplan-Meier curves of the progression-free survival. Progression-free survival was assessed in the whole population $(n=25)$.

1 patient died, and 22 patients were alive. The median follow-up time for PFS was 14.6 months (IQR 13.1-16.2) months. Nineteen of the 24 patients (79.2\%) achieved an overall response, and disease control was achieved in 23 $(95.8 \%)$ patients at 3 months post radiotherapy (table 2, online supplemental figure 1). Among the 23 patients with a confirmed objective response within 12 months post radiotherapy, the median time to response was 2.65 months (IQR 1.88-5.27), and the median duration of response was not reached (not reached to not reached) (table 2). The treatment response remained ongoing in 17 of 25 patients $(68.0 \%)$ at the cut-off point. Overall, all 25 patients $(100.0 \%)$ with at least one post-baseline tumor assessment had a decrease in the size of their target lesions from baseline. The median change from baseline was $-100.0 \%$ (IQR $-71.3 \%$ to $-100.0 \%$ ) (figure 2 ). Five patients had documented disease progression including three local progressions, one regional relapse, and one death. The median PFS was 18.67 months (95\% CI 12.04 to 25.29 months), and the 12-month PFS was $91.8 \%$ (95\% CI $91.7 \%$ to $91.9 \%$ ) (figure 3 ). The median follow-up time for OS was 15.7 months (IQR 13.9-18.0) months, the median OS was not reached, and the 12-month OS was $96.0 \%$ (95\% CI $88.4 \%$ to $100.0 \%$ )

\section{Adverse events}

All 25 patients were included in the safety analysis (table 3 ). The most common AEs were G1-2 fatigue in $22(88.0 \%)$ patients, G1-2 nausea in $19(76.0 \%)$ patients, G1-2 serum creatinine elevation in $13(52.0 \%)$ patients, G1-2 weight loss in $11(44.0 \%)$ patients, G1-2 blood triglyceride elevation in $11(44.0 \%)$ patients, G1-2 hypothyroidism in 8 $(32.0 \%)$ patients, G1-2 pruritus in $8(32.0 \%)$ patients, G1-2 blood cholesterol elevation in $6(24.0 \%)$ patients, G1-2 creatine kinase elevation in $4(16.0 \%)$ patients, G1-2 leukopenia in $4(16.0 \%)$ patients and G1-2 myositis in $4(16.0 \%)$ patients. G3 serum triglyceride elevation was observed in $1(4.0 \%)$ patient, G4 creatine kinase elevation was observed in $1(4.0 \%)$ patient, G3 skin reaction was observed in $2(8.0 \%)$ patients, and G3 mucositis was observed in $1(4.0 \%)$ patient. Regarding late AEs, the most common AEs were G1-2 hearing loss in 18 (72.0\%) patients, G1-2 dry mouth in $18(72.0 \%)$ patients, G1-2 cranial neuropathy in $9(36.0 \%)$ patients, and G1-2 neck tissue damage in 7 (28.0\%) patients. Additionally, we recorded $7(28.0 \%)$ cases of $\geq \mathrm{G} 3$ nasopharyngeal wall necrosis, $3(12.0 \%)$ cases of $\geq \mathrm{G} 3$ nasal bleeding, and 1 $(4.0 \%)$ case of G3 trismus. One patient died due to G5 nasal bleeding attributed to artery blowout.

\section{Exploratory studies}

Nineteen tumor biopsy samples were obtained for IHC staining of PD-L1. Four PD-L1-negative samples (21.1\%) and 15 PD-L1-positive samples $(78.9 \%)$ were identified. PD-L1-positive status was associated with higher CR rates $(86.7 \%$ vs $50.0 \%)$, and PD-L1-positive patients had a better PFS (HR=0.43); however, these differences were not statistically significant (online supplemental tables 3 and 4).

WES results were obtained from 18 patients with rNPC. TMB was determined by analyzing somatic mutations within the coding region of the human genome. TMB was generally low in this study, with a median TMB of 0.72 (IQR 0.28-1.44) mutations per million base pairs. Patients with a non-CR tended to have a higher copy number burden (wGII score) (median, 0.30 vs $0.12, \mathrm{p}=0.056$ ) and TMB (median, 1.25 vs $0.73, \mathrm{p}=0.574$ ) than patients with a CR, while patients with a higher wGII score (HR 2.78, 95\% CI 1.04 to $6.88, \mathrm{p}=0.037$ ) and TMB (HR 2.91, 95\% CI 0.18 to $46.68, \mathrm{p}=0.451)$ tended to have reduced PFS (online supplemental figures 2 and 3 ).

WES identified 4508 genetic alterations, including 370 missense, 30 non-sense, 49 splice site, 52 frameshift mutations, 2131 amplification and 1876 deletions. According to our previous study, the nuclear factor kappa-B (NF-kB), PI3K-AKT, JAK-STAT, and cell cycle pathways were enriched in somatic mutations in $\mathrm{rNPC}_{1}{ }^{18}$ and the DNA mismatch repair pathway was associated with radiotherapy sensitivity. ${ }^{19} 20$ However, no correlation between somatic mutations or these associated pathway alterations and clinical response or improved PFS was found (online supplemental figure 3).

Additional clinical characteristics analyzed for correlation with clinical efficacy included age, sex, Epstein-Barr virus (EBV) DNA copy number, rT stage, rN stage, GTV, GTV Dmin (minimum dose), GTV Dmax (maximum dose), GTV D95 (dose covering 95\% volume) and GTV D50 (median dose). Among the subgroups, early T stages (T2/3) and smaller GTV were associated with a higher CR rate but without a statistically significant difference, while patients with larger GTV had reduced PFS compared with other patients (HR per cc increase 1.04, 95\% CI 1.01 to $1.07, \mathrm{p}=0.021$ ). However, pretreatment EBV DNA copy number was not significantly associated with PFS outcome, while both $\mathrm{rT}$ and $\mathrm{rN}$ stages were also not significant prognostic factors of PFS in these patients. (online supplemental tables 3 and 4, online supplemental figure $4)$. 
Table 3 Adverse events and grade

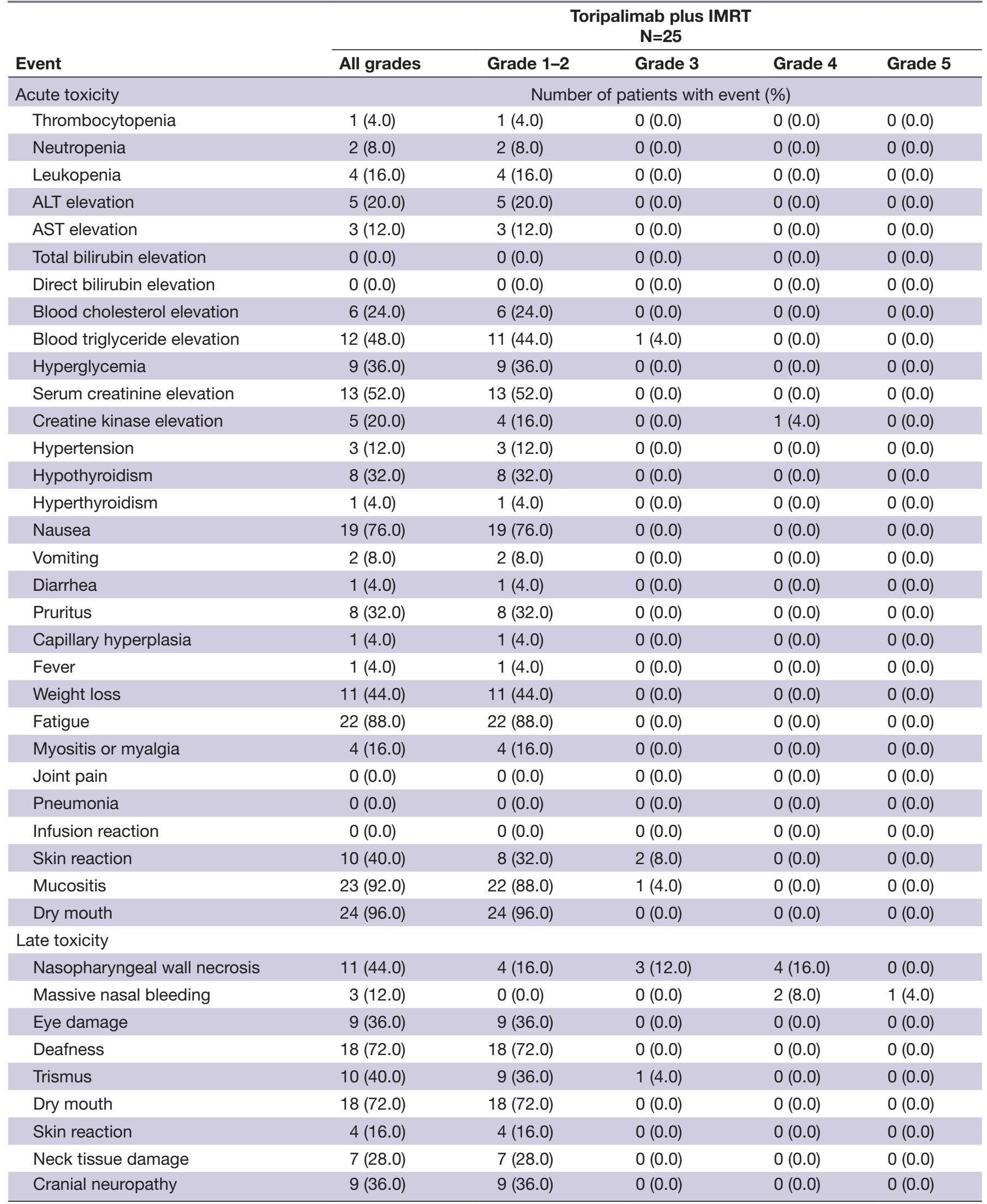

All data are presented as No. (\%).

ALT, Alanine aminotransferase; AST, Aspartate aminotransferase; IMRT, intensity-modulated radiotherapy . 


\section{DISCUSSION}

To our knowledge, this study is the first report to evaluate the combination therapy of an anti-PD-1 antibody and IMRT in patients with NPC. Our results revealed that toripalimab plus IMRT showed promising antitumor activity against unresectable locally rNPC, with a favorable response rate, $\mathrm{PFS}$ outcome and manageable toxicity profile.

The ORR at 3 months post reirradiation was reported to be only $50 \%$ for rNPC, $^{21-24}$ as radiation dose coverage to tumor volumes is often suboptimal due to the close proximity to critical organs at risk. ${ }^{25}$ Although the number of studies on combined chemoradiotherapy in $\mathrm{rNPC}$ has increased in recent years, such as radiotherapy combined with induction and/or concurrent chemotherapy, the role of combination therapy has also remained undefined, with ORR rates of approximately $60 \%$ and 3-year OS rates of $40 \%-50 \%$, while acute side effects have increased significantly, with severe hematological and non-hematological rates of $11.7 \%-42.4 \%$ and $23.5 \%-33.3 \%$, respectively. ${ }^{825-27}$

The main problem of second-course radiotherapy for rNPC is the unsatisfactory local control rate and severe radiation-related injury. Immunotherapy, such as the use of PD-1 inhibitors, has mild side effects and continuous efficacy. Radiotherapy not only has direct killing effects on tumor cells but also overcomes an immunosuppressive tumor microenvironment and promotes antigen presentation. $^{12} 28$ Therefore, radiotherapy combined with PD-1 might be theoretically effective and have lower toxicity. The primary purpose of this trial is to improve the local control rate, especially at the routine assessment point of 3 months after the completion of radiotherapy. Therefore, we prescribed the use of seven cycles of PD-1 therapy, which ended exactly 3 months after the completion of radiotherapy. The ORR findings herein meet the prespecified endpoint for this phase 2 trial. Nineteen $(79.2 \%)$ patients achieved an overall response at 3 months post radiotherapy, which is better than the historical data of $50 \%-65.4 \%{ }^{625}$ which all utilized IMRT with standard fractionated scheme of $60 \mathrm{~Gy}, 27-30$ fractions, five fractions per week as the second-course radiation therapy for rNPC with similar rT stages of $92 \%-100 \%$ of rT3-rT4. This suggests that the efficacy of radiotherapy combined with PD-1 monoclonal antibody treatment is promising.

In our study, toripalimab combined with IMRT was well tolerated, with all patients completing IMRT and $88 \%$ of patients completing seven cycles of toripalimab. Regarding severe acute toxicities, only $4.0 \%-8.0 \%$ patients were observed G3-4 blood triglyceride elevation, creatine kinase elevation, skin reaction or mucositis, respectively, showing that combined PD-1 therapy did not significantly increase the side effects of radiotherapy and systemic side effects. Regarding late AEs, we recorded $11(44.0 \%)$ cases of nasopharyngeal wall necrosis, which is similar to the historical data ${ }^{29} 30 \quad(31.5 \%-40.6 \%)$. However, only $3(12.0 \%)$ patients had G3-4 nasal bleeding because embolization of the affected internal carotid artery was performed for patients with G3-4 nasopharyngeal necrosis, preventing the occurrence of massive nasal bleeding.

Interestingly, copy number burden (wGII score) was observed to be a potential biomarker for PD-1 plus radiotherapy in $\mathrm{rNPC}$, which was consistent with the findings of previous reports on head and neck cancer, prostate cancer, and other cancers demonstrating that copy number burden is a prognostic factor associated with recurrence and death. ${ }^{31-33}$ Finally, we observed that patients with lower GTV had a statistically significant PFS advantage compared with other patients, which also demonstrated that GTV impacted locoregional control in rNPC. 3435

We acknowledge that this study has some limitations. First, this was a single-arm study with no control group for comparison, and thus, selection bias could not be ruled out. Second, the small sample size reduces the certainty of the observed effectiveness.

Our data showed that toripalimab combined with IMRT had promising antitumor activity and manageable toxicity in patients with rNPC. Larger randomized controlled trials are warranted to validate our findings.

\section{Author affiliations}

${ }^{1}$ State Key Laboratory of Oncology in South China, Collaborative Innovation Center for Cancer Medicine, Guangdong Key Laboratory of Nasopharyngeal Carcinoma Diagnosis and Therapy, Sun Yat-sen University Cancer Center, Guangzhou, Guangdong, China

${ }^{2}$ Department of Nasopharyngeal Carcinoma, Sun Yat-sen University Cancer Center, Guangzhou, Guangdong, China

${ }^{3}$ Department of Radiation Oncology, First Affiliated Hospital of Kunming Medical University, Kunming, Yunnan, China

${ }^{4}$ Nasopharyngeal Cancer Center, NanChang Hospital, Sun Yat-sen University (The First Hospital of Nanchang), Nanchang, Jiangxi, China

${ }^{5}$ Department of Biostatistics, School of Public Health, Southern Medical University, Guangzhou, Guangdong, China

Contributors Conception and design: MC, RY. Provision of study materials or patients: YH, RY, Y-LX, XZ, Y-PL, C-YD, Y-LL, C-MG, RZL, QY, ML, RJ, M-XZ, XD, RS, MC. Collection and assembly of data: YH, RY, Z-QW, P-YH, ML, Y-FO. Study supervision: YH, RY, Z-QW, P-YH, ML, Y-FO, MC. Manuscript writing: All authors. Final approval of manuscript: All authors. Accountable for all aspects of the work: All authors. Responsible for the overall content as the guarantor: MC.

Funding Funding was provided by the Key-Area Research and Development of Guangdong Province (2020B1111190001), the National Natural Science Foundation of China (No. 82002857, 81772895, 81874134), the Special Support Program for High-level Talents in Sun Yat-sen University Cancer Center, the Guangzhou Science and Technology Plan Project (202103010001), and the National 'Ten Thousand Talents Program' Science and Technology Innovation Leading Talents (84 000-41180005), Sun Yat-Sen University Clinical Research 5010 Program (No. 2018015), CSCO-JunShi Cancer Immunotherapy Clinical Research Fund (No. Y-JS2019-002).

Competing interests None declared.

Patient consent for publication Not applicable.

Ethics approval The study protocol was approved by the Ethics Committee of the Sun Yat-sen University Cancer Center.

Provenance and peer review Not commissioned; externally peer reviewed.

Data availability statement Data are available upon reasonable request.

Supplemental material This content has been supplied by the author(s). It has not been vetted by BMJ Publishing Group Limited (BMJ) and may not have been peer-reviewed. Any opinions or recommendations discussed are solely those 
of the author(s) and are not endorsed by BMJ. BMJ disclaims all liability and responsibility arising from any reliance placed on the content. Where the content includes any translated material, BMJ does not warrant the accuracy and reliability of the translations (including but not limited to local regulations, clinical guidelines, terminology, drug names and drug dosages), and is not responsible for any error and/or omissions arising from translation and adaptation or otherwise.

Open access This is an open access article distributed in accordance with the Creative Commons Attribution Non Commercial (CC BY-NC 4.0) license, which permits others to distribute, remix, adapt, build upon this work non-commercially, and license their derivative works on different terms, provided the original work is properly cited, appropriate credit is given, any changes made indicated, and the use is non-commercial. See http://creativecommons.org/licenses/by-nc/4.0/.

\section{ORCID iDs}

Rui You http://orcid.org/0000-0001-9362-8144

Mei Lin http://orcid.org/0000-0003-0275-9144

\section{REFERENCES}

1 Cao S-M, Simons MJ, Qian C-N. The prevalence and prevention of nasopharyngeal carcinoma in China. Chin J Cancer 2011;30:114-9.

2 Li YQ, Tian YM, Tan SH, et al. Prognostic model for stratification of radioresistant nasopharynx carcinoma to curative salvage radiotherapy. J Clin Oncol 2018;36:891-9.

3 Lee AWM, Ng WT, Chan JYW, et al. Management of locally recurrent nasopharyngeal carcinoma. Cancer Treat Rev 2019;79:101890.

4 You R, Zou X, Hua Y-J, et al. Salvage endoscopic nasopharyngectomy is superior to intensity-modulated radiation therapy for local recurrence of selected T1-T3 nasopharyngeal carcinoma - a case-matched comparison. Radiother Oncol 2015;115:399-406.

5 Zou X, Han F, Ma W-J, et al. Salvage endoscopic nasopharyngectomy and intensity-modulated radiotherapy versus conventional radiotherapy in treating locally recurrent nasopharyngeal carcinoma. Head Neck 2015;37:1108-15.

6 Lee VHF, Kwong DLW, Leung T-W, et al. Hyperfractionation compared to standard fractionation in intensity-modulated radiation therapy for patients with locally advanced recurrent nasopharyngeal carcinoma. Eur Arch Otorhinolaryngol 2017;274:1067-78.

7 Leong YH, Soon YY, Lee KM, et al. Long-Term outcomes after reirradiation in nasopharyngeal carcinoma with intensity-modulated radiotherapy: a meta-analysis. Head Neck 2018;40:622-31.

8 Guan Y, Liu S, Wang H-Y, et al. Long-Term outcomes of a phase II randomized controlled trial comparing intensity-modulated radiotherapy with or without Weekly cisplatin for the treatment of locally recurrent nasopharyngeal carcinoma. Chin J Cancer 2016;35:20.

9 Hsu C, Lee S-H, Ejadi S, et al. Safety and antitumor activity of pembrolizumab in patients with programmed Death-Ligand 1-positive nasopharyngeal carcinoma: results of the KEYNOTE-028 study. J Clin Oncol 2017;35:4050-6.

10 Ma BBY, Lim W-T, Goh B-C, et al. Antitumor activity of nivolumab in recurrent and metastatic nasopharyngeal carcinoma: an international, multicenter study of the Mayo clinic phase 2 Consortium (NCI-9742). J Clin Oncol 2018;36:1412-8.

11 Fang W, Yang Y, Ma Y, et al. Camrelizumab (SHR-1210) alone or in combination with gemcitabine plus cisplatin for nasopharyngeal carcinoma: results from two single-arm, phase 1 trials. Lancet Oncol 2018;19:1338-50.

12 Reits EA, Hodge JW, Herberts CA, et al. Radiation modulates the peptide repertoire, enhances MHC class I expression, and induces successful antitumor immunotherapy. J Exp Med 2006;203:1259-71.

13 Gupta A, Probst HC, Vuong V, et al. Radiotherapy promotes tumorspecific effector CD8+ T cells via dendritic cell activation. $J$ Immunol 2012;189:558-66.

14 Powell SF, Gold KA, Gitau MM, et al. Safety and efficacy of pembrolizumab with chemoradiotherapy in locally advanced head and neck squamous cell carcinoma: a phase lb study. J Clin Oncol 2020;38:2427-37.

15 Jabbour SK, Berman AT, Decker RH, et al. Phase 1 trial of pembrolizumab administered concurrently with chemoradiotherapy for locally advanced non-small cell lung cancer: a nonrandomized controlled trial. JAMA Oncol 2020;6:848-55.

16 Zhang Y, Chen L, Hu G-Q, et al. Gemcitabine and cisplatin induction chemotherapy in nasopharyngeal carcinoma. $N$ Engl J Med 2019;381:1124-35

17 Dudek AZ, Liu LC, Gupta S, et al. Phase Ib/Il clinical trial of pembrolizumab with bevacizumab for metastatic renal cell carcinoma: BTCRC-GU14-003. J Clin Oncol 2020;38:1138-45.

18 You R, Liu Y-P, Lin D-C, et al. Clonal mutations activate the NF- $\kappa B$ pathway to promote recurrence of nasopharyngeal carcinoma. Cancer Res 2019;79:5930-43.

19 Bucci B, D'Agnano I, Amendola D, et al. Myc down-regulation sensitizes melanoma cells to radiotherapy by inhibiting MLH1 and MSH2 mismatch repair proteins. Clin Cancer Res 2005;11:2756-67.

20 Kim W-ju, Vo QN, Shrivastav M, et al. Aberrant methylation of the ATM promoter correlates with increased radiosensitivity in a human colorectal tumor cell line. Oncogene 2002;21:3864-71.

21 Chan OSH, Sze HCK, Lee MCH, et al. Reirradiation with intensitymodulated radiotherapy for locally recurrent $\mathrm{T} 3$ to $\mathrm{T} 4$ nasopharyngeal carcinoma. Head Neck 2017;39:533-40.

22 Qu S, Lin S, Tham IWK, et al. Intensity-modulated radiation therapy in the salvage of locally recurrent nasopharyngeal carcinoma. Int $J$ Radiat Oncol Biol Phys 2012;83:676-83.

23 Zheng X-K, Ma J, Chen L-H, et al. Dosimetric and clinical results of three-dimensional conformal radiotherapy for locally recurrent nasopharyngeal carcinoma. Radiother Oncol 2005;75:197-203.

24 Oksüz DC, Meral G, Uzel O, et al. Reirradiation for locally recurrent nasopharyngeal carcinoma: treatment results and prognostic factors. Int J Radiat Oncol Biol Phys 2004;60:388-94.

$25 \mathrm{Ng} \mathrm{W}-\mathrm{T}$, Ngan RKC, Kwong DLW, et al. Prospective, multicenter, phase 2 trial of induction chemotherapy followed by BioChemoradiotherapy for locally advanced recurrent nasopharyngeal carcinoma. Int J Radiat Oncol Biol Phys 2018;100:630-8.

26 Kong F, Zhou J, Du C, et al. Long-term survival and late complications of intensity-modulated radiotherapy for recurrent nasopharyngeal carcinoma. BMC Cancer 2018;18:1139.

27 Hua Y-J, Han F, Lu L-X, et al. Long-term treatment outcome of recurrent nasopharyngeal carcinoma treated with salvage intensity modulated radiotherapy. Eur J Cancer 2012;48:3422-8.

28 Frey B, Gaipl US. Radio-immunotherapy: the focused beam expands. Lancet Oncol 2015;16:742-3.

29 Chen H-yan, Ma X-mei, Ye M, et al. Effectiveness and toxicities of intensity-modulated radiotherapy for patients with locally recurrent nasopharyngeal carcinoma. PLoS One 2013;8:e73918.

30 Han F, Zhao C, Huang S-M, et al. Long-Term outcomes and prognostic factors of re-irradiation for locally recurrent nasopharyngeal carcinoma using intensity-modulated radiotherapy. Clin Oncol 2012;24:569-76.

31 Dubot C, Bernard V, Sablin MP, et al. Comprehensive genomic profiling of head and neck squamous cell carcinoma reveals FGFR1 amplifications and tumour genomic alterations burden as prognostic biomarkers of survival. Eur J Cancer 2018;91:47-55.

32 Hieronymus $\mathrm{H}$, Murali $\mathrm{R}$, Tin A, et al. Tumor copy number alteration burden is a pan-cancer prognostic factor associated with recurrence and death. Elife 2018;7. doi:10.7554/eLife.37294. [Epub ahead of print: 0409 2018].

33 Hieronymus H, Schultz N, Gopalan A, et al. Copy number alteration burden predicts prostate cancer relapse. Proc Natl Acad Sci U S A 2014;111:11139-44.

34 Lin Y-H, Huang T-L, Chien C-Y, et al. Pretreatment prognostic factors of survival and late toxicities for patients with nasopharyngeal carcinoma treated by simultaneous integrated boost intensitymodulated radiotherapy. Radiat Oncol 2018;13:45.

35 lacovelli NA, Cicchetti A, Cavallo A, et al. Role of IMRT/VMATBased dose and volume parameters in predicting 5-year local control and survival in nasopharyngeal cancer patients. Front Oncol 2020;10:518110. 